\title{
Three-Dimensional Modeling of Microsphere Contact/Impact with Smooth, Flat Surfaces
}

\author{
W. Cheng, R. M. Brach, and P. F. Dunn \\ Particle Dynamics Laboratory, Department of Aerospace and Mechanical Engineering, \\ University of Notre Dame, Notre Dame, Indiana
}

\begin{abstract}
A three-dimensional (3D) simulation model of a microsphere in contact with a nominally-smooth, flat surface is established. The model is based on Hertzian contact stresses, an idealized ring force distribution of adhesion, nonlinear damping, and friction. Although originally developed for the study of oblique impact, the model also is shown to describe the motion of a microsphere in sustained contact with a flat surface, including nonlinear normal oscillatory motion in the presence of sliding and rolling. Nonlinear, normal oscillatory motion is illustrated using conventional phaseplane techniques. Methods for the determination of damping coefficients associated with normal motion from impact experiments are discussed. The significance and modeling of rolling resistance and implications of asymmetric contact stress distributions are presented. Simulations show that energy exchange between translation and rotation can play an important role during oblique impact. The effects of complex initial conditions on the rebound and capture of a microsphere are significant as are the effects of contact friction. Results show that the inability to measure and the failure to account for rotational velocitiesin experimental measurements can limit the intepretation of the results.
\end{abstract}

\section{INTRODUCTION}

Microsphere contact/impact with surfaces has many applications and much theoretical significance. The modeling of microsphere contact/impact is important in predicting particle deposition on and rebound from surfaces. The results can be used in contamination monitoring and control, especially in environmental particle sampling and collection. On the other hand, the issue of microsphere contact/impact is closely related to advances in micro-scale contact mechanics, for example, in the understanding and modeling of various surface forces. The results can be useful in an even wider range of areas. Microsphere

Received 12 September 2001; accepted 14 March 2002.

This research was supported in part during its early phase by the Center for Indoor Air Research (contract 96-06) and the Electric Power Research Institute (contract RP 8034-03).

Address correspondence to Patrick F. Dunn, 107 Hessert Center, Notre Dame, IN 46556. E-mail: patrick.f.dunn.1@undedu contact with flat surfaces relates to the interpretation of the output of surface force apparatus such as Atomic Force Microscope (AFM) (Burnham et al. 1997). More recent applications are found in the area of Micro Electro Mechanical Systems (MEMS) and nano-technology, which involves micrometer-and nanometer-sized components (Rollot et al. 1999). Also, the microsphere contact/impact model is fundamental in granular flow and geomechanics modeling. The Hertzian elastic contact theory for spheres is used with discrete element approaches for the constitutive model of granular materials. Fundamental aspects in the modeling of discrete element systems are summarized by Cundall and Hart (1992). More applications can be easily found for single and multimicrosphere contact/impact models.

Classical studies on the contact/impact problem may be divided into 3 chronological stages. In the first stage, models were restricted to rigid bodies. The basic ideas were based on Newton's third law and Coulomb's friction law for rigid bodies. Some of the results are still used widely today. In the second stage, local elasticity over the contact area was included. The geometry and deformation of a contact body was assumed such that a closed-form solution could be found by using the available mathematical and mechanical tools. Application of Hertz's work on the static contact problem of 2 spheres is regarded as a milestone in the field (Hertz 1881, 1882). Following this work, many researchers studied contact problems between elastic bodies of different shapes under different circumstances with or without friction. One of the leading contributions is the Johnson-Kendall-Roberts (JKR) model (Johnson 1985). Examples can also be found in Goldsmith (1960) and Gladwell (1980). The third stage is numerical simulation. Computational methods including finite-element methods are widely used in simulations in contact mechanics in which contact bodies are discretized as the collection of finite elements. More complicated geometry, boundary conditions, and loading can be considered. The contact bodies may deform in an arbitrary way. Examples can be found in Zhong (1993) and Quesnel et al. (1998). Except for the advantages of third-stage methods, the modeling of surface interactive forces such as adhesion and friction on the contact 
boundary still depends on the results of the second stage. The roughness of the nominal smooth surface is still modeled by the contact of microspherical asperities with or without friction and adhesion. The sphere contact/impact model remains the fundamental case for interface analysis.

Most studies have focused on the normal attraction acting between solid surfaces (detailed explanations can be found in Israelachvili and Tabor (1972)). The theoretical adhesive contact area of spherical surfaces under elastic deformation is derived by Johnson et al. (1971) and Derjaguin et al. (1975). The tangential friction problem for oblique contact/impact still is not fully solved. Some effort has been made to model the tangential adhesion contact to extend the JKR model for the case of tangential loading (Savkoor and Briggs 1977; Thornton 1991). Criteria for tangential separation have been proposed, including that a tangential force is capable of causing normal separation of the surface, which is called "peeling." Skinner and Gane (1972) and Ando et al. (1995) measured the relationship between the dry friction and the normal attraction forces between surfaces under micro-loads when tangential motion occurs. Several impact models have been developed recently to predict the oblique rebound velocity of the microsphere (Brach and Dunn 1995; Dahneke 1975; Wall et al. 1990; Tsai et al. 1991; Xu and Willeke 1993).

Experiments and numerical simulations have focused on the accurate prediction of the rebound velocities of spheres in planar motion. However, three-dimensional (3D; degrees of freedom) measurements and simulations have not been reported. Yet the full nonlinear nature of the problem can only be visualized through 3D simulations with a wide range of initial conditions. 3D simulations reflect the properties of the simulation model and generate useful information needed to improve understanding of contact/impact problems. Another reason for 3D simulations is that in reality microparticle impacts are almost always 3D with 6 degrees of freedom. In this paper a 3D simulation model for microsphere impact with a flat surface is developed. Then an experimental data fitting method is introduced to determine the damping coefficients required as known parameters in the simulation.

The focus of this paper is the oblique impact of microspheres. However, some aspects of dynamics of established contact are also covered. The model for friction and rolling resistance in this paper is different from those used by other researchers such as Wang (1990) and Ziskind et al. (1997). Some existing models of rolling are fundamentally limited when external or inertia forces are not included in the balance of moments, leading to incomplete moments for rolling. In the current simulation the surfaces of the microsphere and the substrate are considered to be smooth. Even though all surfaces are rough at the microscopic level, there are compelling reasons to assume that the surfaces are smooth. In reality roughness has scale levels. In order to keep a nominal flat geometry, roughness must have a much smaller wavelength and height than the curvature or the radius of the contact area. Any surface irregularity with approximately the same order in height and wavelength as the contact area is considered as a large geometry problem and contradicts the assumption of a single sphere Hertzian contact with a flat surface. A result of this reasoning is that no moment is generated from the normal contact. Another difference relates to the tangential loading or tangential inertia force. In Ziskind et al. (1997), because the tangential loading is assumed to be much smaller than the dry friction force (in other words, sliding does not occur), the tangential contact was modeled as a linear elastic spring. For impact, there is little or no experimental evidence to indicate that tangential elasticity plays a significant role. On the other hand, ample evidence exists (Brach et al. 2000) that a tangential contact force composed primarily of dry friction is appropriate.

Some argue that in particle removal, when external forces such as gravity are weak, an energy method based on vibrational resonance may be used. This may not be a productive approach because the concept of resonance is linear, whereas Hertzian contact and sphere vibration is a nonlinear phenomenon. The approach in this paper considers the nonlinear properties of the normal contact through Brach and Dunn's model. The possibility of resonance is discussed.

Several cases of 3D oblique impact are simulated to identify the effects of changes in initial conditions and surface friction on the energy dissipation and rebound velocities. The sensitivity of parameters and some potential sources of errors in experiments are identified through the numerical simulations. The results show that 3D simulation and nonlinear analysis are important tools to understand the mechanism of particle impact and deposition with surfaces.

\section{SIMULATION MODEL}

The current simulation model was proposed by Brach and Dunn (1995) and was based on Hertzian contact theory under adhesion loading. Figure 1 shows the coordinate system pertaining to the 3D impact between a microsphere and a flat surface where $\dot{n}<0$ initially. Typically the contact radius, $a$, is small compared to the sphere radius, $r$, and the radius of curvature of the contact area. Because the particle is small, the elastic force and the adhesion force are the dominant forces. Gravity is negligible and not considered. Energy is dissipated through the material and adhesion damping.

According to Newton's second law, the equation of normal motion is

$$
m \ddot{n}=F_{H}+F_{H D}+F_{A}+F_{A D} .
$$

The first term on the right side of Equation (1) is the Hertzian elastic force, $F_{H}$. It is calculated from the Hertzian theory

$$
F_{H}=\sqrt{r} K(-n)^{3 / 2}, \quad n \leq 0,
$$

where $n$ is the relative displacement of the mass center and $n \leq 0$ during contact. The original radius of the microsphere is $r$. The effective material stiffness is $K$, which is defined by the following equations:

$$
K=\frac{4}{3 \pi\left(k_{1}+k_{2}\right)},
$$




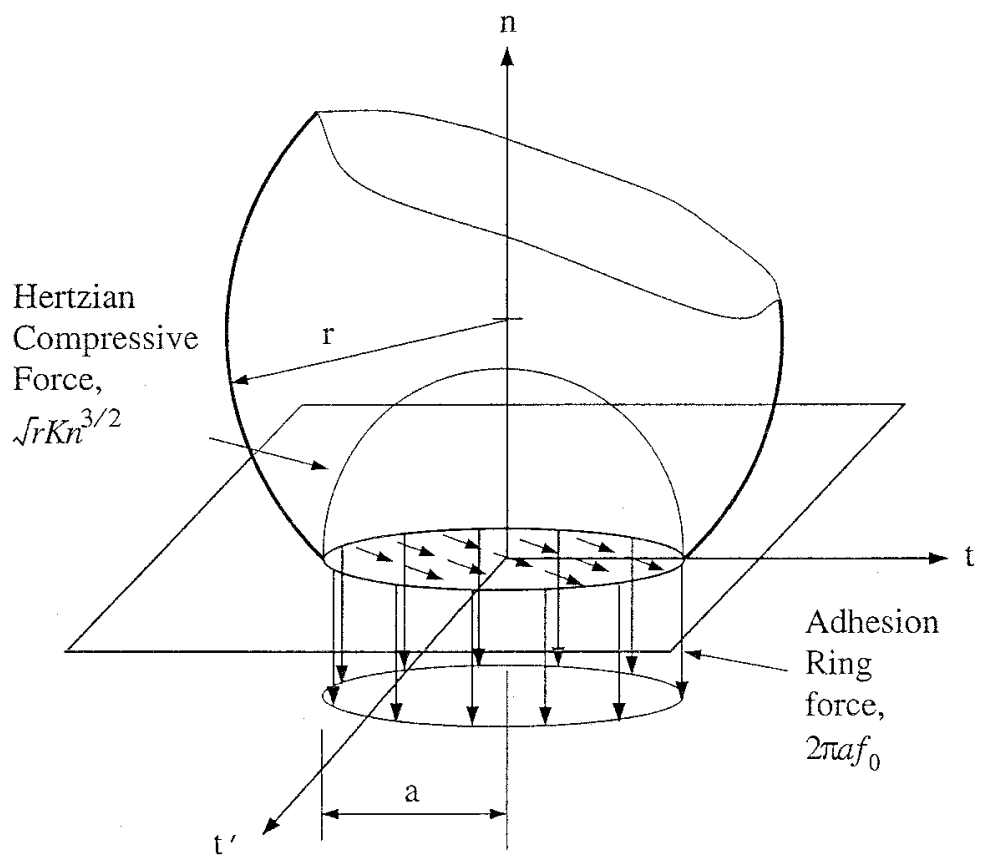

Figure 1. Schematic of a sphere in contact with a flat surface.

and

$$
k_{i}=\frac{\left(1-v_{i}^{2}\right)}{\pi E_{i}}, \quad i=1,2,
$$

where $v_{i}$ and $E_{i}$ are Poisson's ratio and Young's modulus, respectively, for the sphere and the substrate material. The adhesion force, $F_{A}$, in Equation (1) is modeled as a ring force acting on the periphery of the circular contact area, where the intensity of the adhesion ring force is $f_{0}$ :

$$
F_{A}=-2 \pi a f_{0} .
$$

As predicted by Hertzian theory, the contact radius, $a$, is

$$
a=\sqrt{-r n} .
$$

The calculation of the intensity of the adhesion ring force, $f_{0}$, uses a relationship between Brach and Dunn's model and the JKR model. It is assumed that both models produce the same contact radius at the equilibrium position. Thus the following equation was used to calculate $f_{0}$ (Li et al. 1999):

$$
f_{0}=\left(\frac{9}{2 \pi} K r w_{A}^{2}\right)^{1 / 3}
$$

In Equation (7), $w_{A}$ is the combined surface energy between the sphere and the surface materials. $w_{A}$ can be obtained through Dupré's equation:

$$
w_{A}=F_{1}+F_{2}-F_{12},
$$

[8] The damping coefficients, $C_{H}$ and $C_{A}$, can be put into where $F_{1}$ and $F_{2}$ are surface free energies of body 1 and body 2 , respectively, and $F_{12}$ is the interfacial free energy. In reality, the surface tensions of the 2 bodies, $\gamma_{1}$ and $\gamma_{2}$, and the interfacial tension, $\gamma_{12}$, are used instead:

$$
w_{A}=\gamma_{1}+\gamma_{2}-\gamma_{12}
$$

The interfacial tension can be estimated by

$$
\gamma_{12}=\gamma_{1}+\gamma_{2}-2 \Phi_{12}\left(\gamma_{1} \gamma_{2}\right)^{1 / 2}
$$

Note that the interaction parameter, $\Phi_{12}$, is not exactly at unity. Practically, however, it is near unity. The uncertainty in using $\Phi_{12}=1$ is $<2 \%$. This small correction is a second-order effect that is negligible in the current model. Thus the surface energy can be estimated as follows:

$$
w_{a} \cong 2 \sqrt{\gamma_{1} \gamma_{2}}
$$

The values of $f_{0}$ for different cases used in the simulation are listed in Table 1. Dynamic damping is included using firstpower velocity dependence for the material damping, $F_{H D}$, and the adhesion damping, $F_{A D}$ :

$$
F_{H D}=-F_{H} C_{H} \dot{n},
$$

and

$$
F_{A D}=F_{A} C_{A} \dot{n} .
$$


Table 1

The basic parameters for numerical simulation

\begin{tabular}{ccccccccc}
\hline Reference & $r(\mu m)$ & $\begin{array}{c}\text { Density } \\
\left(\mathrm{kg} / \mathrm{m}^{3}\right)\end{array}$ & $K\left(\times 10^{9} \mathrm{~Pa}\right)$ & $f_{0}(N / \mathrm{m})$ & $\zeta_{A}$ & $\zeta_{H}$ & $f$ & Descriptions \\
\hline Wall et al. (1990) & $1.29-3.45$ & 1350 & 1.776 & $5.340-7.390$ & 29.0 & 0.50 & 0.15 & Figure 3 \\
Wall et al. (1990) & 3.45 & 1350 & 1.776 & 7.422 & 29.0 & 0.50 & 0.15 & Figures 5, 7-12 \\
Wall et al. (1990) & 3.45 & 1350 & 1.776 & 7.422 & 29.0 & 0.50 & $0.10-0.90$ & Figures 13-14 \\
\hline
\end{tabular}

nondimensional forms:

$$
\begin{aligned}
& \zeta_{A}=C_{A}\left(\frac{r^{3} K \sqrt{v_{n}}}{m}\right)^{2 / 5}, \\
& \zeta_{H}=C_{H}\left(\frac{r^{3} K \sqrt{v_{n}}}{m}\right)^{2 / 5},
\end{aligned}
$$

where $v_{n}$ is a reference velocity, typically the initial normal velocity. The nondimensionaldamping coefficients are determined through an experimental data fitting procedure that will be introduced in the following section. Two tangential directions in the Cartesian coordinate system, $t$ and $t^{\prime}$, are considered to include the "off-plane" spin and sliding in the simulation. The equation of motion in the $t$ direction is

$$
m \ddot{t}=F_{t} .
$$

The equation of motion in the $t^{\prime}$ direction is

$$
m \ddot{t^{\prime}}=F_{t^{\prime}}
$$

A form of Coulomb friction with a coefficient $f$ is used for the tangential friction. At the beginning and the end stage of contact, the normal force is negative due to the fact that for small displacement, $n$, the adhesion force is larger than the Hertzian elastic force. Thus a friction for "pulling" force contact has to be modeled. In the current simulation model, an assumption is made that the contact is tangentially rigid and the tangential deformation is neglected. The tangential friction force is assumed to be proportional to the magnitude of the "pulling" force based on the fact that in the "snap-on" and "snap-off" procedure, the particle is in "contact" with the substrate surface and the friction still exists due to the relative motion at the interface. This friction model agrees with the measurement results of Skinner and Gane (1972). The frictional force has components $F_{t}$ and $F_{t^{\prime}}$ that oppose the relative tangential contact velocity and are proportional to the resultant normal force, $F_{n}$. Except for an isolated special case, the initial relative tangential contact velocity is nonzero for oblique microsphere impacts. This means that a friction force exists initially. If during contact friction causes sliding to end, a sphere continues to move under the condition of pure rolling and sliding cannot reoccur.

It should be mentioned that the behavior of tangential friction in the presence of adhesion is still not fully understood, particu- larly when tangential elastic deformation is significant. An elastic model and an extension of the JKR model in the tangential direction have been discussed (Ziskind et al. 2000; Savkoor and Briggs 1977; Thornton 1991). However, for impact problems in which the initial relative tangential contact velocity is large, separation likely occurs over the whole contact area. The transition from shear traction to sliding friction can be neglected. The model is tangentially rigid in the current simulation. Even though this condition is simplified, it appears suitable for the simulation of impact problems.

As a result of the tangential friction, moments, torque, and rotation are also produced about the mass center. The microsphere is considered as a rigid body in modeling the spin and rotation. The spin about the normal axis, $n$, is described in the following rigid-body equation:

$$
I \dot{\omega}_{n}=2 \pi \int_{0}^{a} f\left|p_{n}\right| \rho^{2} d \rho=-\frac{75 \pi f a F_{n}}{64 r^{2}},
$$

where $I$ is the centroidal moment of inertia of the sphere and $p_{n}$ is the normal contact pressure over the contact area. The torsional moment caused by friction in Equation (18) is calculated by the integration of Coulomb friction about the center of the contact area. The equations of rotation about the tangential axes are the following:

$$
\begin{aligned}
I \dot{\omega}_{t} & =-r F_{t^{\prime}}, \\
I \dot{\omega}_{t^{\prime}} & =r F_{t},
\end{aligned}
$$

In order to describe and compare the simulation results, several coefficients are defined. The restitution coefficient, $e_{n}$, is the ratio of the magnitude of rebound (subscript, $r$ ) to initial (subscript, $i$ ) normal (subscript, $n$ ) velocities:

$$
e_{n}=\frac{v_{r_{n}}}{v_{i_{n}}}
$$

The tangentialimpulse ratios, $\mu_{t}$ and $\mu_{t^{\prime}}$, are defined as the ratio of tangential impulse to normal impulse after impact in $t$ and $t^{\prime}$ directions:

$$
\begin{aligned}
\mu_{t} & =\frac{v_{t_{r}}-v_{t_{i}}}{v_{n_{r}}-v_{n_{i}}}, \\
\mu_{t^{\prime}} & =\frac{v_{t_{r}^{\prime}}-v_{t_{i}^{\prime}}}{v_{n_{r}}-v_{n_{i}}} .
\end{aligned}
$$


In the absence of adhesion, the upper limit of the tangential impulse ratio is the Coulomb friction coefficient, $f$. However, in the current nonlinear model, when the "snap-on" and "snap-off" period is long, the tangential impulse ratio can be larger than $f$. The normalized total kinetic energy loss coefficient, $K_{t}$, is the percentage of total energy loss (total energy means the summation of translational and rotational kinetic energy) after impact:

$$
K_{t}=\frac{m\left(v_{i}^{2}-v_{r}^{2}\right)+I\left(\omega_{i}^{2}-\omega_{r}^{2}\right)}{\left(m v_{i}^{2}+I \omega_{i}^{2}\right)} .
$$

The normalized translational kinetic energy loss coefficient, $K_{L}$, is defined as the ratio of translational kinetic energy loss to the initial translational kinetic energy:

$$
K_{L}=\frac{v_{i}^{2}-v_{r}^{2}}{v_{i}^{2}} .
$$

A reason for defining 2 different kinetic energy loss terms is because, experimentally, the rotational kinematics typically are not measured. Consequently, experimental kinetic energy loss values reported in the past correspond to Equation (25), yet the total kinetic energy loss is given by Equation (24). The simulationallows both to be determined (Brach and Dunn 1996).

\section{ROLLING RESISTANCE}

The equations for rolling in the current simulation (Equations (19) and (20)) are different from those used by other researchers. It is suggested (for example, see Wang (1990)) that a moment, or couple, due to adhesion over the contact area produces a resistance to rotation. The magnitude of this resistance is estimated as the product of a "pull-off" force and the contact radius. Although this theory has been commonly accepted (Ziskind et al. 1997), it can overestimate rolling resistance significantly. Figure 2 illustrates schematically the conditions that occur when a microsphere in contact with a surface rolls in the presence of adhesion. Both the Hertzian and adhesion contact forces develop an asymmetry, causing their lines of action to move away from the normal axis, $n$. In the case of the adhesion force, this asymmetry is due to the leading edge of the contact area establishing new contact and the trailing edge pulling away. This causes a moment, $M_{t^{\prime}}$, that resists rolling where

$$
M_{t^{\prime}}=x_{a} F_{a}+x_{h} F_{h} .
$$

The force $F_{a}$ includes the adhesion force, $F_{A}$, and the corresponding adhesion damping, $F_{A D}$, and the force $F_{h}$ includes the Hertzian force, $F_{H}$, and the corresponding Hertzian damping,

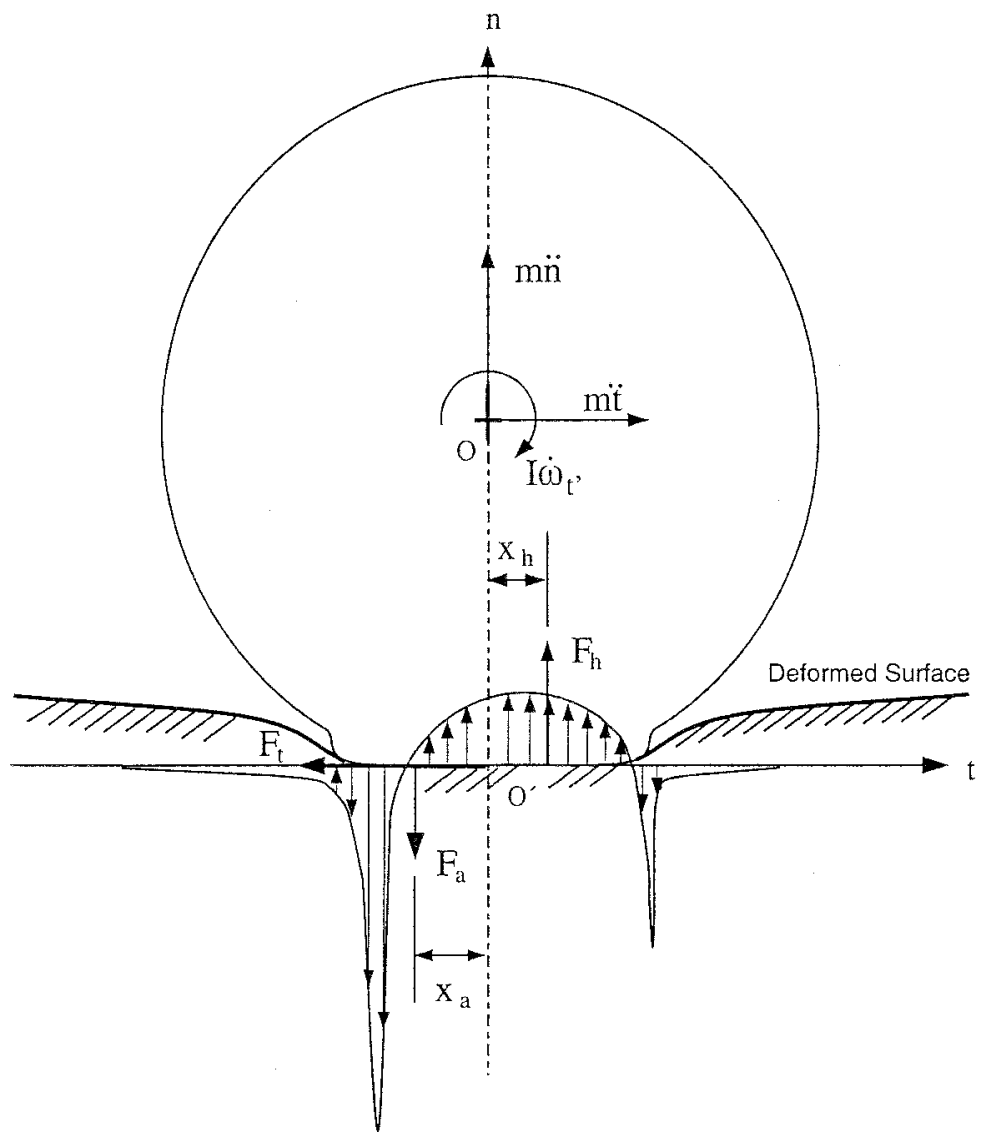

Figure 2. Schematic for the analysis of the rolling resistance. 
$F_{H D}$. The distances $x_{a}$ and $x_{h}$ are the offsets of the lines of action of the adhesion and Hertzian forces, respectively. This phenomenon was modeled for the process of impact by Brach et al. (1999b), where it was found that rolling resistance has a negligible effect during impact and attachment of microspheres. Some evidence exists to indicate that this rolling resistance may also be negligible for particle resuspension (Dunn et al. 2001). For this reason it is not included in the model presented in the previous section.

\section{DETERMINATION OF THE DAMPING COEFFICIENTS}

The Runge-Kutta-Gill (R-K-G) method was used to solve the previously described governing equations. In the $3 \mathrm{D}$ simulation, 4 parameters are required: the effective material stiffness, $K$, the adhesion line force, $f_{0}$, the nondimensional Hertzian damping coefficient, $\zeta_{H}$, and the nondimensional adhesion damping coefficient, $\zeta_{A}$. The estimation of the material stiffness and the adhesion line force are introduced in the previous section. The damping coefficients, $\zeta_{H}$ and $\zeta_{A}$, can be determined by the fitting of the 2D microsphere impact data. For each set of experimental conditions, an optimal set of $\zeta_{H}$ and $\zeta_{A}$ is found to fit the coefficient of restitution, $e_{n}$. Figure 3 shows an example of the least-square regression of the experimental data of Wall et al. (1990). After fitting, the values of $\zeta_{H}$ and $\zeta_{A}$ are fixed and the set with the least-square error are selected for 3D simulation. The values used in the simulation are listed in Table 1.

\section{NONLINEAR DYNAMICS OF NORMAL OSCILLATORY MOTION}

Most, if not all, previous studies of microsphere impact have considered only before-and-after contact conditions. Numerical simulation offers a way to understand the dynamics of particle impact and the effects of parameters during contact as well. This is particularly pertinent after attachment, when it occurs. Ziskind et al. (2000) estimate a natural frequency of vertical oscillation using a simplified contact model. The system is linearized near the equilibrium position and damping is neglected. However, nonlinear systems do not possess a single natural frequency because of a strong dependence on initial conditions. Even for a linear system, an over-damped system has no natural frequency. The model covered in this paper can be used to simulate vertical oscillatory motion of an attached microsphere.

Because the tangential and rotational motion is driven by the normal contact for a Coulomb frictional system, proper representation of the nonlinear dynamics of the normal process is crucial. By changing the coordinate system, $x=-n$, the equation of motion at the normal direction can be rewritten as

$$
m \ddot{x}=-\sqrt{r} K x^{3 / 2}\left(1+C_{H} \dot{x}\right)+2 \pi f_{0} \sqrt{r} x^{1 / 2}\left(1-C_{A} \dot{x}\right) .
$$

Using the same symbols for the displacement of the mass center, $x$, and the time, $t$, the nondimensional form of Equation (27) is

$$
\ddot{x}=-x^{3 / 2}\left(1+C_{1} \dot{x}\right)+x^{1 / 2}\left(1-C_{2} \dot{x}\right) .
$$

The following length, $X$, and time scales, $T$, are used in nondimensionalization:

$$
X=\frac{2 \pi f_{0}}{K},
$$

$$
T=\frac{m}{\sqrt{r} K} X^{-1 / 2}=\frac{m}{\sqrt{2 \pi r f_{0} K}} .
$$

The length scale, $X$, is also the equilibrium displacement, and the time scale, $T$, is set so that the Hertzian force is at the same

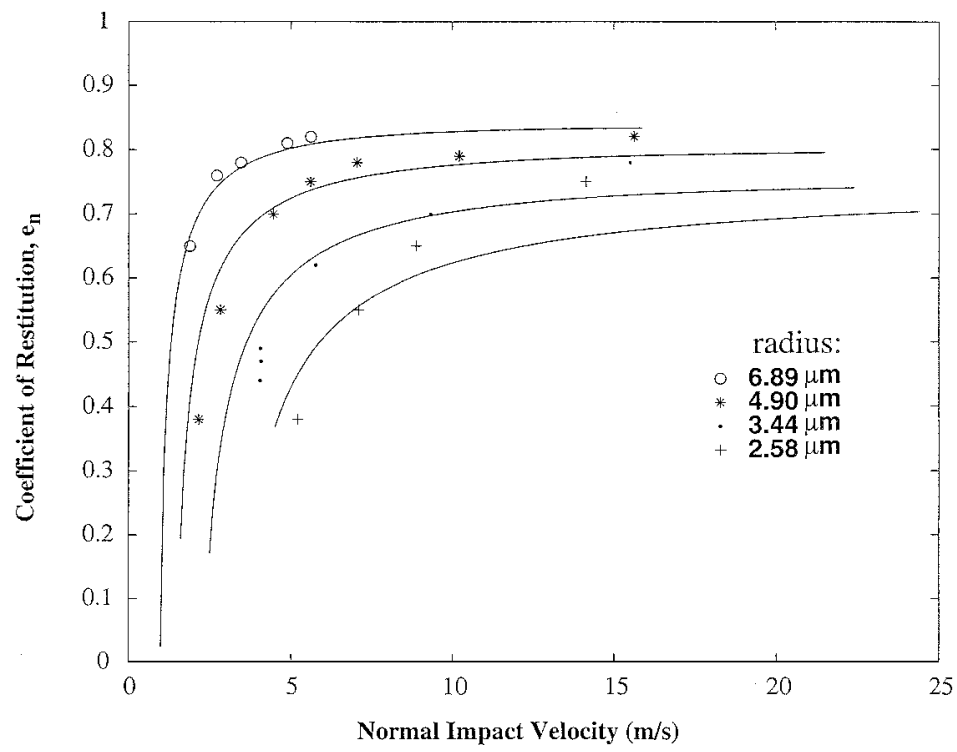

Figure 3. Numerical simulation results (solid lines) and Wall et al. data (1990), $\zeta_{A}=29.0, \zeta_{H}=0.50$. 


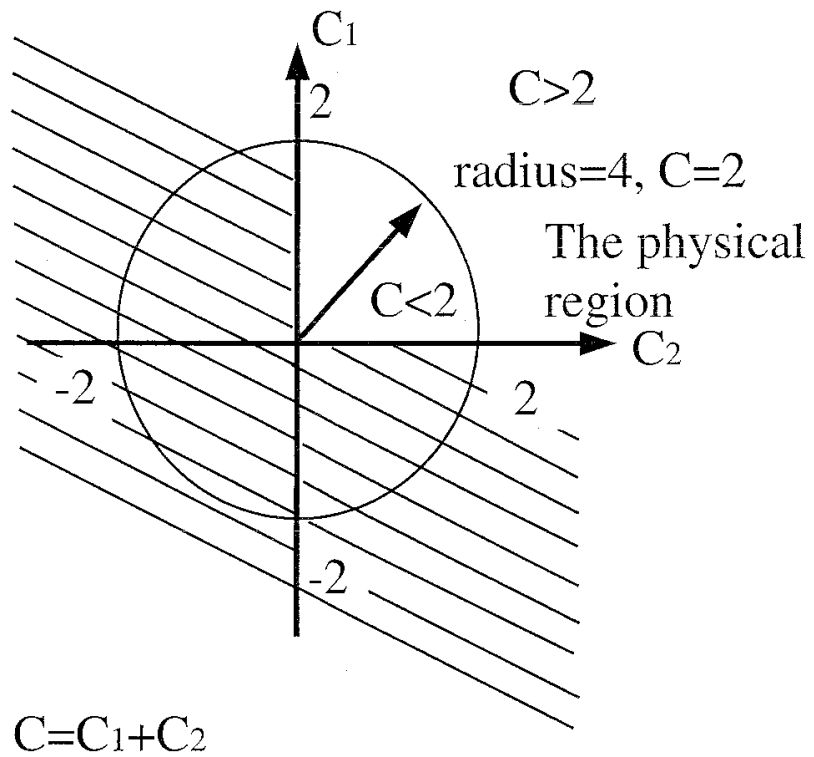

Figure 4. Bifurcation on the parameter plane.

order of the adhesion force, which is true for adhesive impact problems. The nondimensional damping parameters, $C_{1}$ and $C_{2}$ (different from $\zeta_{H}$ and $\zeta_{A}$ ), are

$$
C_{1}=C_{H} \frac{X}{T}=C_{H} \sqrt{\frac{r}{K}} \frac{\left(2 \pi f_{0}\right)^{3 / 2}}{m},
$$

and

$$
C_{2}=C_{A} \frac{X}{T}=C_{A} \sqrt{\frac{r}{K}} \frac{\left(2 \pi f_{0}\right)^{3 / 2}}{m} .
$$

The system form of Equation (28) is

$$
\left\{\begin{array}{c}
\dot{x}=y \\
\dot{y}=-x^{3 / 2}\left(1+C_{1} y\right)+x^{1 / 2}\left(1-C_{2} y\right)
\end{array}\right\} .
$$

The Jacobian of Equation (33) is

$$
\begin{aligned}
& J(x, y) \\
& =\left(\begin{array}{cc}
0 & 1 \\
-\frac{3}{2} x^{1 / 2}\left(1+C_{1} y\right)+\frac{1}{2} x^{-1 / 2}\left(1-C_{2} y\right) & -x^{3 / 2} C_{1}-x^{1 / 2} C_{2}
\end{array}\right) .
\end{aligned}
$$

Because translation of the coordinates, $x$ and $y$, does not affect the Jacobian, $J$, the frequency of the solution at any point $(x, y)$ in the phase portrait is the imaginary part of the eigenvalue of the Jacobian, $J$. Because the eigenvalues of $J$ are functions of both $x$ and $y$ (the displacement and the impact velocity), it is clear that no single natural frequency exists for the contact/impact problem based on the model with adhesion and damping.
Equation (33) has 2 equilibrium points. The nonlinear properties of the contact system can be studied at the equilibrium position of the adhesion contact $(x=1, y=0)$. The Jacobian at 1,0 is

$$
J(1,0)=\left(\begin{array}{cc}
0 & 1 \\
-1 & -C
\end{array}\right)
$$

where $C=C_{1}+C_{2}$. The eigenvalues of $J(1,0)$ are

$$
\lambda=\frac{-C \pm \sqrt{C^{2}-4}}{2} .
$$

A bifurcation on the parameter plane occurs with a critical point, $C=2$. Figure 4 shows the parameter plane. When $C>2$, both eigenvalues are real. It corresponds to the over-damped system and no frequency is possible at all. When $C=2$, a double nonzero eigenvalue appears; this corresponds to a critically-damped case in which the frequency is zero. When $C<2$, a damped oscillation occurs. The frequency is $\sqrt{4-C^{2}}$ and the point $(1,0)$ is a spiral sink because the real part of the eigenvalue is negative (represents an amplitude decay). Physically, these different properties of the equilibrium position correspond to 3 kinds of solutions in the simulation: rebound (spiral sink, large incident velocity, Figure 5a), capture with oscillation (spiral sink, low incident velocity, Figure 5b), and capture without oscillation (pure sink, Figure 5c).

The nonlinear analysis of the nondimensional system can be projected back to the dimensional system. In the dimensional sphere contact/impact problem, 3 types of numerical solutions from the 3D simulation model are shown in Figure 5 that correspond to the experiments of Wall et al. (1990) for microspheres with a radius of $3.45 \mu \mathrm{m}$. 


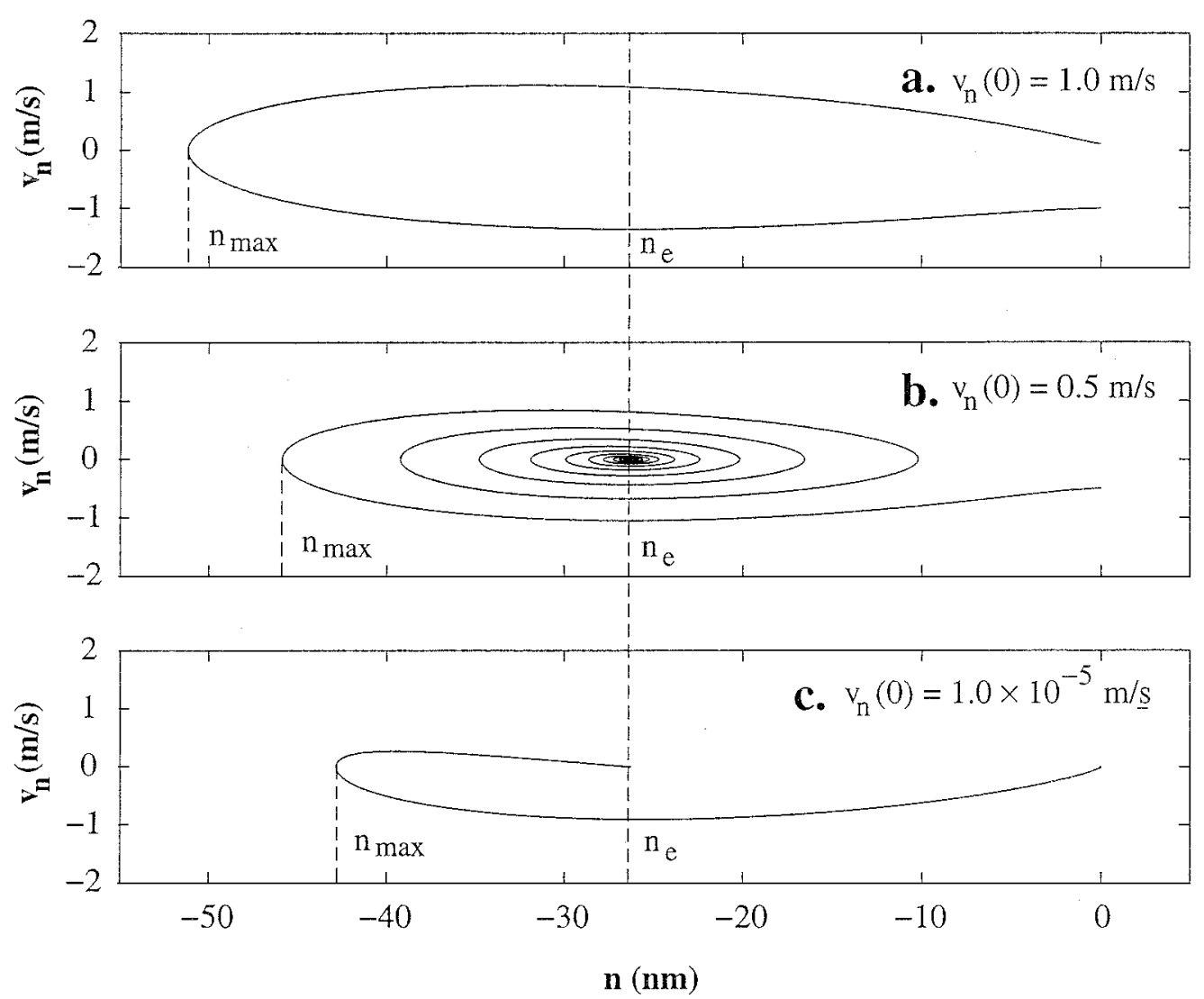

Figure 5. Phase portraits for variations of incident velocities.

When the forces on the right side of Equation (1) balance, the microsphere is attracted to the surface and reaches the equilibrium position. The velocity at the equilibrium position is zero, and the displacement at the equilibrium position is the equilibrium displacement, $n_{e}=X$. Physically, a microsphere sitting on the surface with no external force is at its static equilibrium position or an equilibrium position can be approached after a microsphere is attached on the surface.

Define a constant, $C_{T}$, where $C_{T}=C_{H}+C_{A}$, a total damping coefficient. The property of the equilibrium position is determined by the following conditions:

1. When $C_{T}<2\left(2 \pi f_{0}\right)^{-5 / 4} r^{-1 / 4} K^{3 / 4} m^{1 / 2}$, the equilibrium position is a spiral sink. The solution around the equilibrium position is a damped oscillation with an asympotic frequency:

$$
\omega_{f}=\frac{\sqrt{4 X^{1 / 2}-C_{T}^{2} \frac{X^{2}}{T} X^{3}}}{2 T} .
$$

It can be shown from Equations (37), (14), and (15) that $\omega_{f}$ depends not only on the material properties and the radius of the sphere but also on the initial velocity $v_{n}$. $\omega_{f}$ cannot be considered as a single natural frequency of the system because the Hertzian damping and adhesion damping forces are nonlinear. The damped oscillationnear the equilibrium position identifies the energy dissipation mechanism for particle capture. It also indicates a potential mechanism for particle resuspension.

2. When $C_{T}=2\left(2 \pi f_{0}\right)^{-5 / 4} r^{-1 / 4} K^{3 / 4} m^{1 / 2}$, the solution around the equilibrium position is a critical damping oscillation.

3. When $C_{T}>2\left(2 \pi f_{0}\right)^{-5 / 4} r^{-1 / 4} K^{3 / 4} m^{1 / 2}$, the system is an over-damped system. The equilibrium point is a sink. The sphere is captured by the surface without oscillation.

If all the material parameters are fixed and the damping coefficients, $C_{H}$ and $C_{A}$, have the form as shown in Equations (15) and (14), the property of the solution is determined by the incident velocity. The critical velocity, $v_{c}$, is defined as

$$
v_{c}=2^{5 / 4} \pi^{25 / 4} f_{0}{ }^{25 / 4} r^{-19 / 4} K^{-23 / 4} m^{-1 / 2}\left(\zeta_{H}+\zeta_{A}\right)^{5} .
$$

The critical velocity for the case in Figure 5 is $v_{c}=2.25 \times$ $10^{-5} \mathrm{~m} / \mathrm{s}$. When the incident velocity is larger than the critical velocity (see Figures $5 \mathrm{a}$ and $5 \mathrm{~b}$ ), the equilibrium point is a spiral sink. In the case of $v_{n}(0)=1.0 \mathrm{~m} / \mathrm{s}$, the maximum displacement, $n_{\text {max }}$, is far away from the equilibrium displacement, $n_{e}$. Thus the inertia of the sphere in the first cycle is large enough to overcome the attraction of the spiral sink and the sphere rebounds 




Figure 6. Schematic of the incident angles.

after impact. In Figure 5a, the impact begins with zero normal displacement and a negative velocity, $-1 \mathrm{~m} / \mathrm{s}$, and ends with a zero normal displacement and a positive impact velocity. In the case of $v_{n}(0)=0.5 \mathrm{~m} / \mathrm{s}$, Figure $5 \mathrm{~b}$, the incident velocity is not large enough and $n_{\max }$ is near $n_{e}$. The microsphere falls into the path of the spiral sink and is captured. The velocity and displacement then form a damped cyclic path near the final equilibrium point. This phase portrait indicates a vibrational contact. The frequency of vibration near the equilibrium position in Figure $5 \mathrm{~b}$ according to Equation (37) is $2.0 \times 10^{7} \mathrm{~Hz}$. The final velocity of the particle is zero, but the final static equilibrium displacement is negative. This type of motion exists whether the initial conditions are due to impact or external forces.

When the incident velocity is smaller than the critical velocity (the case $v_{n}(0)=1.0 \times 10^{-5} \mathrm{~m} / \mathrm{s}$, Figure $5 \mathrm{c}$ ), the equilibrium point is a pure sink and the particle is captured and retained by the surface without oscillation. Detailed theoretical analysis offers the accurate expressions for the capture velocity as a function of material parameters (Cheng et al. 2000).

\section{EFFECTS OF INITIAL CONDITIONS}

In order to understand the energy dissipation and the relationship between rotation and tangential motions, 3D oblique impacts are simulated. Figure 6 is a schematic diagram illustrating the geometry and the incident direction. The values for the initial conditions used in the example cases are listed in Table 2 and the values of the corresponding basic parameters in Table 1. The normalized translational kinetic energy loss coefficient, $K_{L}$, normalized total kinetic energy loss coefficient, $K_{t}$, restitution coefficient, $e_{n}$, and tangential impulse ratio, $\mu$, are compared by variations of the initial conditions over the range listed in Table 2. The magnitude of the initial velocitiesin Table 2 is represented by $v$.

Table 2

The initial conditions used to simulate their effects on 3D impact

\begin{tabular}{lll}
\hline Case number & \multicolumn{1}{c}{ Conditions } & \multicolumn{1}{c}{ Descriptions } \\
\hline No. 2.1 & \multicolumn{1}{c}{$v=0.2 \rightarrow 10 \mathrm{~m} / \mathrm{s}, \alpha=0^{\circ} \rightarrow 30^{\circ}$} & Figures 7, 8 \\
No. 2.2 & $v=0.2 \rightarrow 10 \mathrm{~m} / \mathrm{s}, \alpha=0^{\circ}, \omega_{t^{\prime}}=0 \rightarrow 1.8 \times 10^{5} \mathrm{rad} / \mathrm{s}$ & Figures 9, 10 \\
No. 2.3 & $v=0.2 \rightarrow 10 \mathrm{~m} / \mathrm{s}, \alpha=0^{\circ} \rightarrow 30^{\circ}, \omega_{t}=2.9 \times 10^{5}$ & Figures 11, 12 \\
No. 2.4 & $v=0.2 \rightarrow 10 \mathrm{~m} / \mathrm{s}, \alpha=\theta=0^{\circ}, \omega_{t}=\omega_{t^{\prime}}=2.9 \times 10^{5} \mathrm{rad} / \mathrm{s}, f=0 \rightarrow 0.9$ & Figure 13 \\
No. 2.5 & $v=0.2 \rightarrow 10 \mathrm{~m} / \mathrm{s}, \alpha=\theta=0^{\circ}, \omega_{t}=\omega_{t^{\prime}}=2.9 \times 10^{6} \mathrm{rad} / \mathrm{s}, f=0 \rightarrow 0.9$ & Figure 14 \\
\hline
\end{tabular}




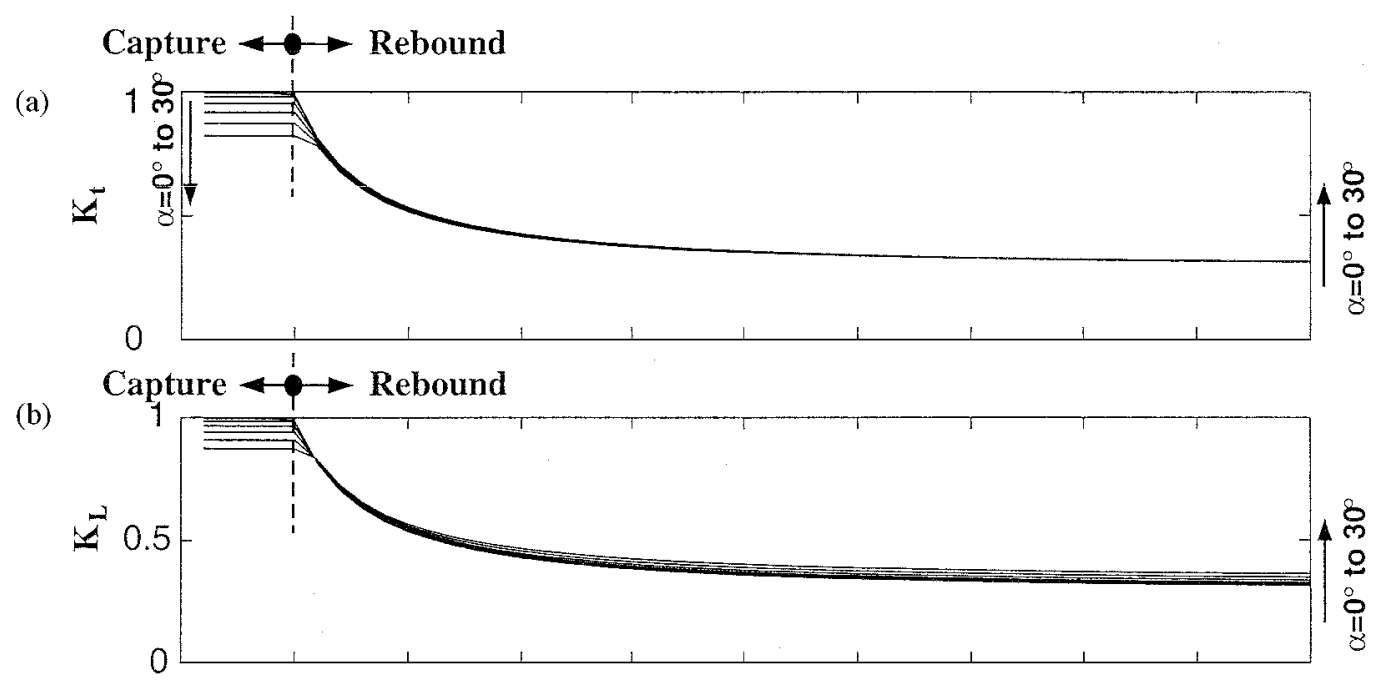

(c)

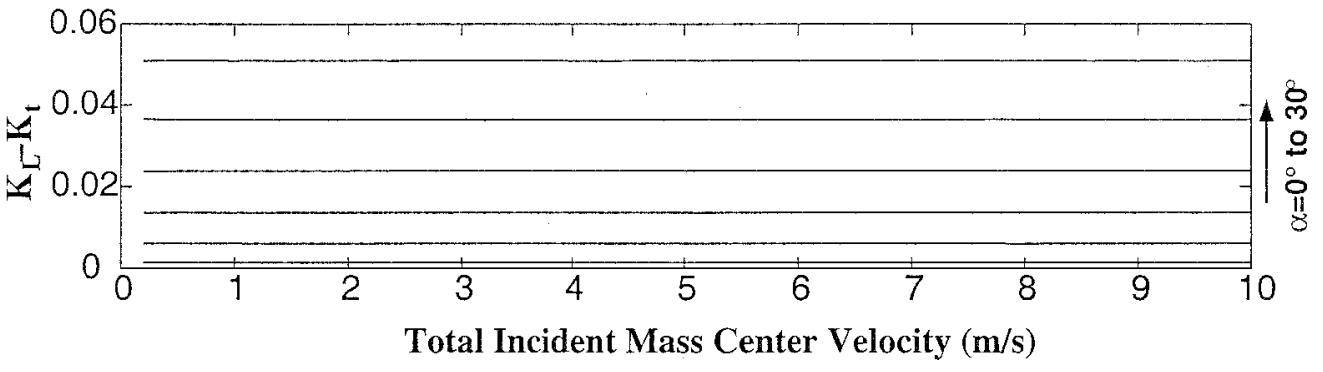

Figure 7. Energy loss for case No. 2.1 ( $\alpha=5^{\circ}$ increment).

An oblique impact is complicated because the friction is nonlinear and rolling and sliding of the microsphere are difficult to predict. In order to understand the energy loss by friction and energy transport between rotation and tangential translation, the total energy loss coefficients and translational energy loss coefficients are plotted as a series of curves related to the incident angles and incident velocities. The curves show the sensitivity of the energy loss to the incident velocities and the incident angles.

The sensitivity of energy loss to the magnitude of incident velocities and impact angles is shown in Figure 7. For the same impact angle $\alpha$, by decreasing the magnitude of the impact velocity, both $K_{L}$ and $K_{t}$ increase. For the same magnitude of impact velocity, when the impact angle, $\alpha$, increases, $K_{L}$ and $K_{t}$ increase. When the incident velocity is smaller than the capture velocity, the particle is captured by the surface and the energy loss is the maximum. In Figure 7, the normalized energy losses for captured particles are constant. For oblique impacts, following capture, microspheres continue to roll. In the current simulation, rolling friction is neglected and the energy loss ratios for captured particles are $\leq 1.0$. In reality, because of rolling friction all of the initial kinetic energy eventually would be dissipated. The difference between $K_{L}$ and $K_{t}$ shows that the translational energy loss, $K_{L}$, is larger than the total energy loss, $K_{t}$, in the oblique impact without initial rotational velocities. For nonzero incident angles, in addition to the energy dissipation, part of the translational energy transforms into rotational energy. It is also clear in Figure 7 that the increase of the impact angle, $\alpha$, causes the increase of the difference, $K_{L}-K_{t}$. This reveals that for a shallow impact angle (a large $\alpha$ ), the rotational energy becomes significant and measurement of rotational velocities is crucial in an experiment.

Figure 8 shows the sensitivity of the tangential impulse ratio, $\mu$, and the coefficient of restitution, $e_{n}$, to impact velocities and impact angles. It is clear that $\mu$ can be sensitive to the increase in approach angle. This means that near capture a small error in the measurement of the impact angle, $\alpha$, can cause a large difference between the experimental and simulation results in tangential velocities. A high accuracy in angle measurement is needed in oblique impact experiments. The numerical simulations show that $e_{n}$ drops at low incident velocities and when the incident velocity is smaller than the capture velocity, $e_{n}$ is zero. The effects of the incident angle on $e_{n}$ are significant at low velocities (depending on the physical properties of the sphere and the surface). This means that the normal rebound velocity is more sensitive to the incident angles for low velocity oblique impacts.

The effects of initial rotational velocities on normal impacts are shown in Figures 9 and 10. Figure 9 shows that neither $K_{L}$ nor $K_{t}$ is sensitive to the initial rotational velocities for the range of initial angular velocities chosen. In order to make a difference 
(a)

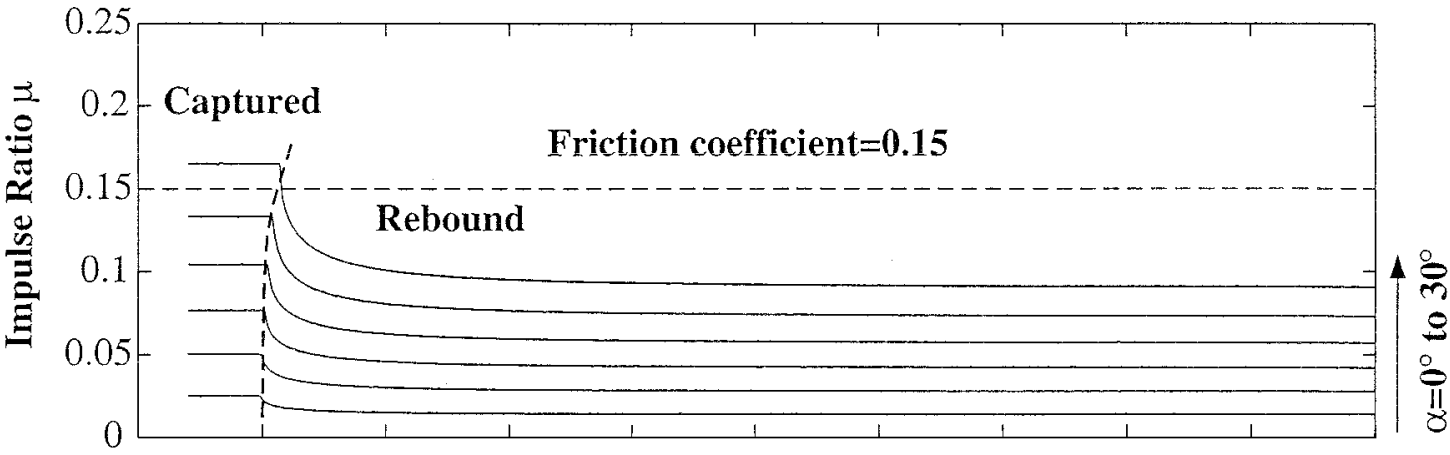

(b)

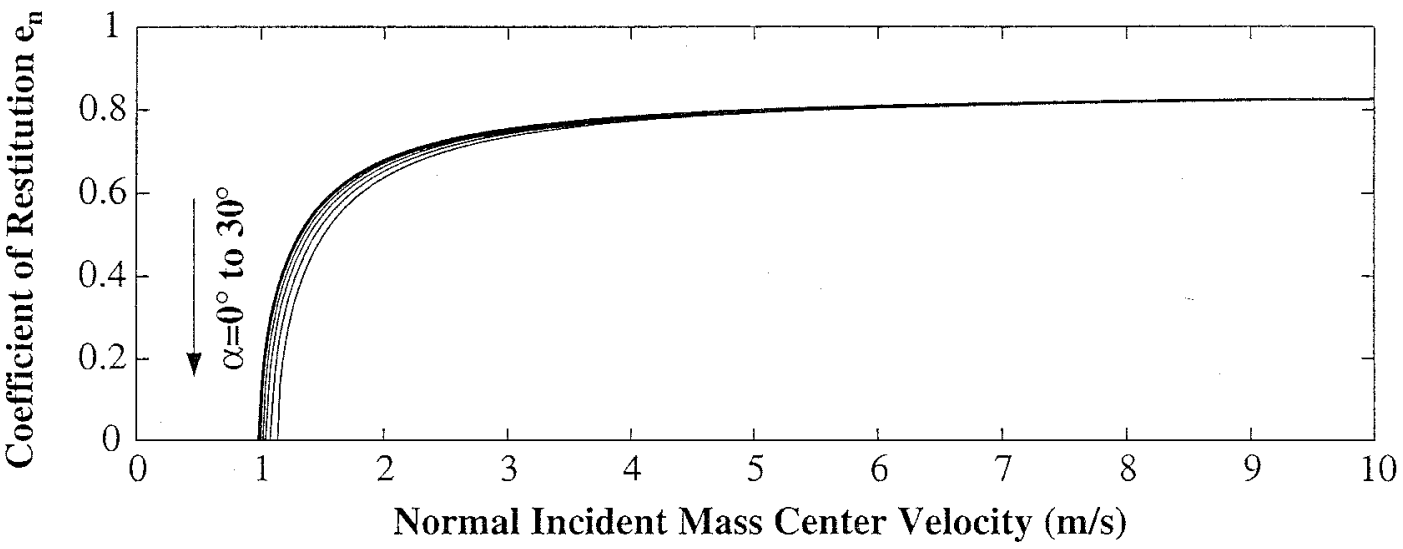

Figure 8. $e_{n}$ and $\mu$ for case No. 2.1 ( $\alpha=5^{\circ}$ increment).

(a)

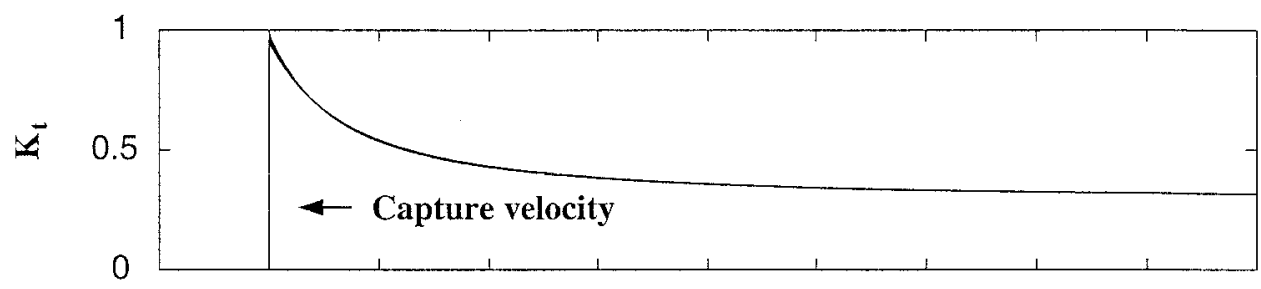

(b)

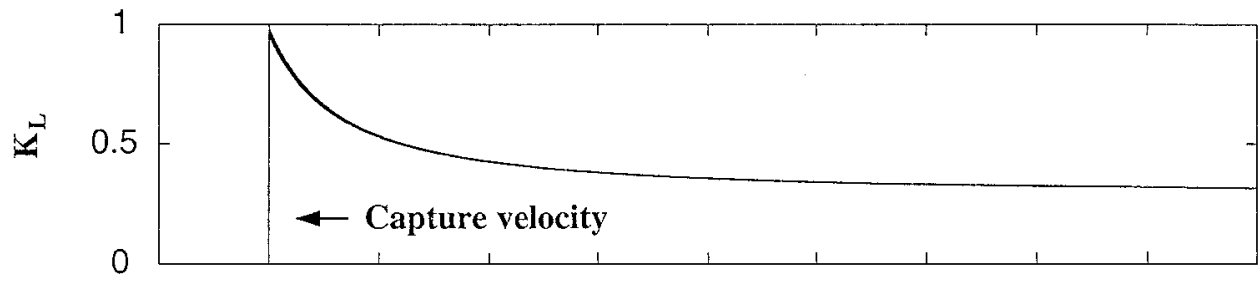

(c)

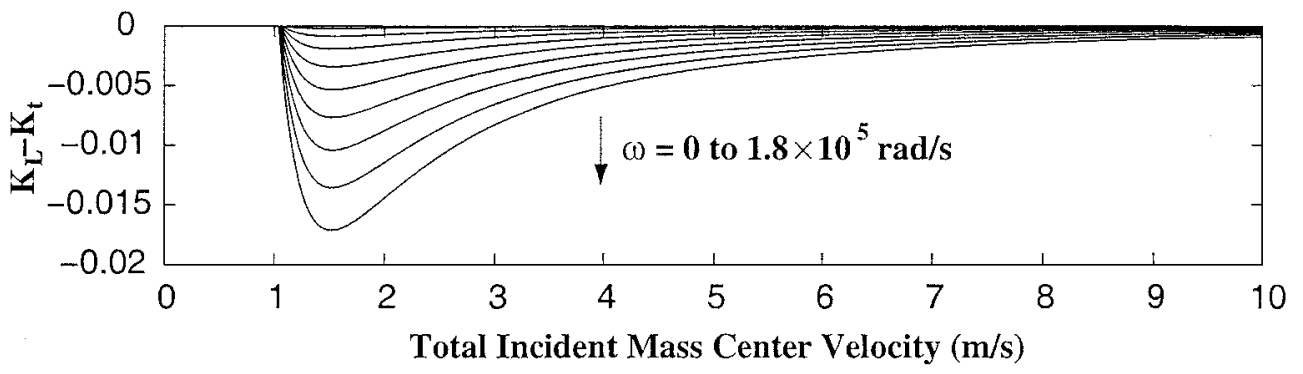

Figure 9. Energy loss for case No. 2.2. 
(a)

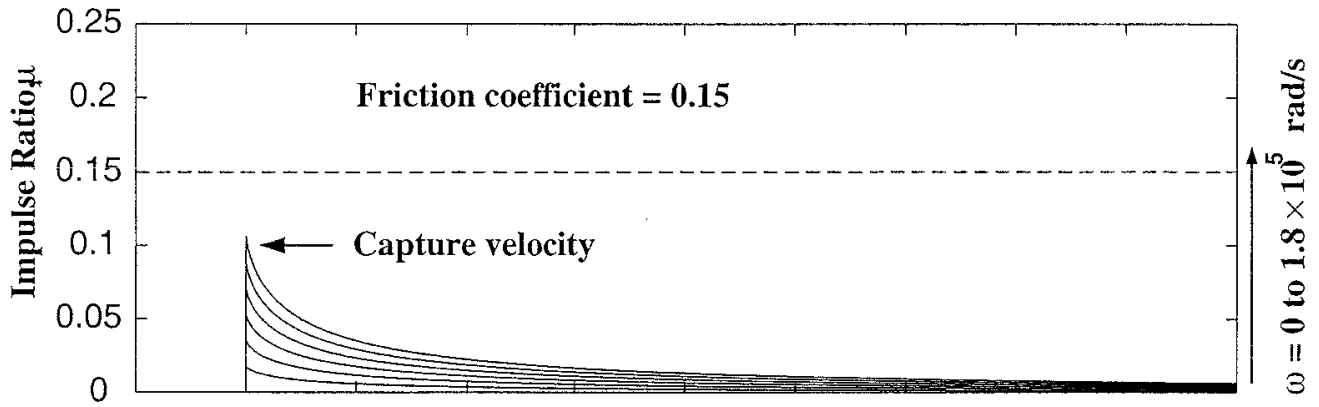

(b)

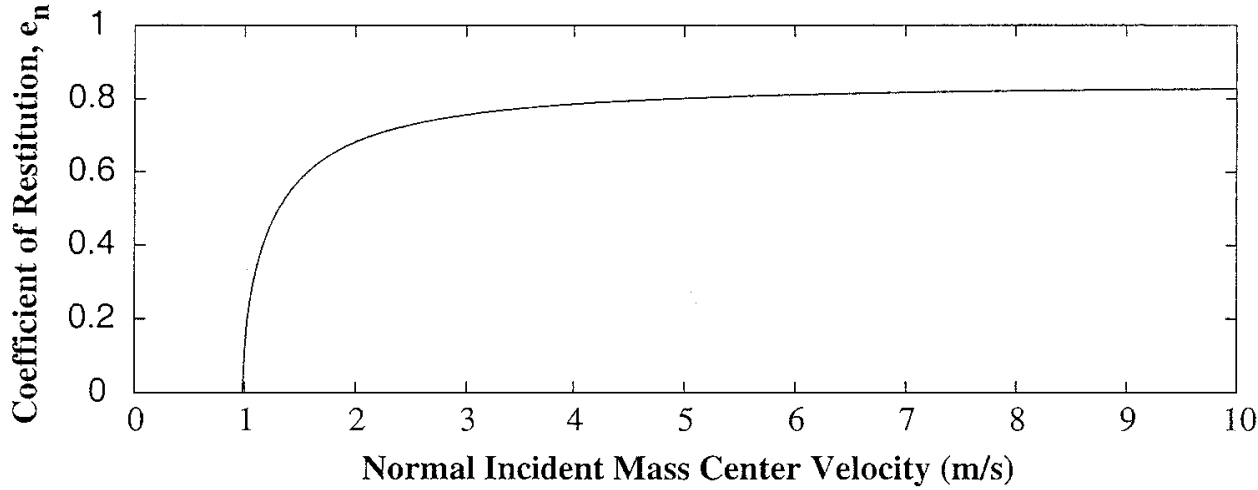

Figure 10. $e_{n}$ and $\mu$ for case No. 2.2.

in $K_{L}$ and $K_{t}$, an initial rotational velocity on the order of $10^{5} \mathrm{rad} / \mathrm{s}$ is needed. However, in reality, the rotation of the particle may still be important because it is possible to have such high initial rotational velocities for microspheres. For a radius of $6.89 \mu \mathrm{m}$, a velocity of $1.0 \mathrm{~m} / \mathrm{s}$ at the center of mass, and zero velocity at the contact area, the rotational velocity is $2.9 \times$ $10^{5} \mathrm{rad} / \mathrm{s}$. The difference, $K_{L}-K_{t}$, is associated with the magnitude of incident velocities. The magnitude of $K_{L}-K_{t}$ is larger at low incident velocities. The rotational energy dissipation is important for low velocity impact. In Figure 9, the values of $K_{L}-K_{t}$ are negative because the initial rotational velocity of the particle increases the initial tangential contact velocity of the particle and influences the translational energy loss.

Figure 10 shows the effects of initial particle rotational velocity on $\mu$ and $e_{n}$. The particle rotation has no effect on the normal impact, which is characterized by the coefficient of restitution, $e_{n}$. All the values for $e_{n}$ under different initial angular velocities fall onto a single curve. However, the effects of the particle rotation on the tangential impulse ratio, $\mu$, are quite obvious. In order to explain the experimental results of particle impact, the rotational rate of the particle, which is extremely difficult to measure for small particles, must be included. The values for energy losses and $\mu$ are not calculated when the incident velocities are smaller than the capture velocity in Figures 9 and 10.

Figures 11 and 12 show the combined effect of initial "offplane" spin, $\omega_{t}$, and the incident angle, $\alpha$, on the impact results. The initial rotational velocity, $\omega_{t}$, is $2.9 \times 10^{5} \mathrm{rad} / \mathrm{s}$. From
Figure 11, by changing the value of $\alpha$, both $K_{L}$ and $K_{t}$ show similar behavior as in Figures 7 and 9. The difference $K_{L}-K_{t}$ is the combination of Figures 7 and 9 . At low velocities $(<4 \mathrm{~m} / \mathrm{s}$ in the simulated cases), the effects of initial rotation are more significant. The values of $K_{L}-K_{t}$ are negative. At high velocities, the effect of incident angle becomes more important. The values of $K_{L}-K_{t}$ are positive. In Figure 12, the changing of $\alpha$ has significant effects on the tangential impact along the $t$ direction, which is represented by $\mu_{t}$. It has less effect on the normal restitution coefficient, $e_{n}$, and tangential impulse ratio along the "off-plane" direction, $\mu_{t^{\prime}}$. The variations of $e_{n}$ and $\mu_{t^{\prime}}$ are caused by the variation of normal impact velocity at different incident angles. Near the capture velocity, the tangential impulse ratio, $\mu_{t^{\prime}}$, is much larger than the friction coefficient, $f$, because of the significant "snap-on" and "snap-off" effects. This result indicates that the coupled effects of adhesion and friction can be significant near capture.

Simulation results also show that the friction coefficient affects the tangential motion and rotation of the microsphere. The effects are closely related to the initial rotation and the relative tangential velocities as shown in Figures 13 and 14. It is reasonable that the initial spin has no effect on the restitution coefficient. In Figure 13, because the initial rotational velocity is small for all the values of the friction coefficients shown in Table 2, the particle is rolling at the end of impact (except in the case of zero friction). All the curves for $\mu_{t}$ and $\mu_{t^{\prime}}$ fall onto a single curve. However, in Figure 14, the initial rotational velocity 
(a)



(b)

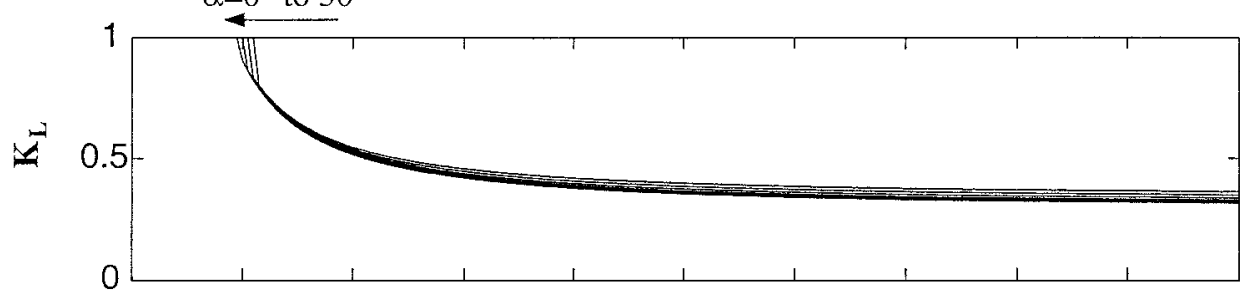

(c)

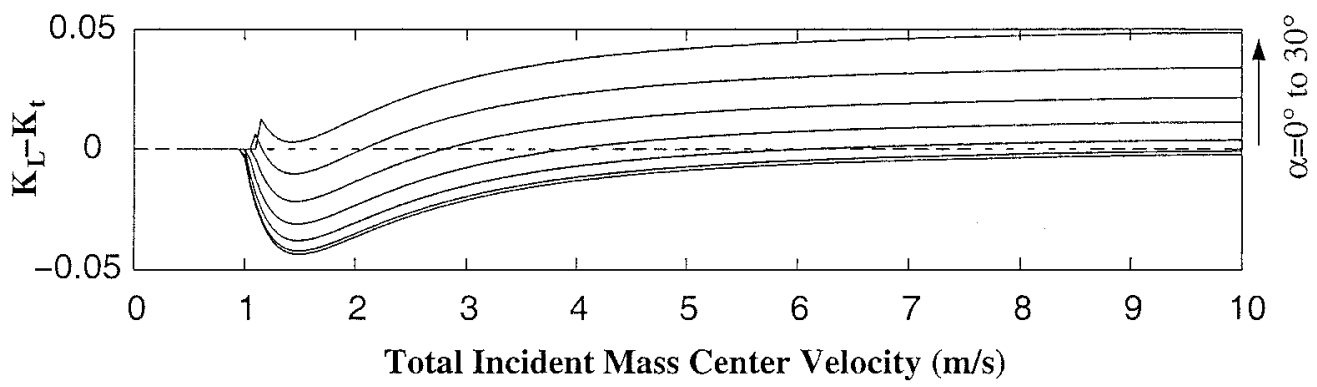

Figure 11. Energy loss for case No. 2.3 ( $\alpha=5^{\circ}$ increment).

(a)

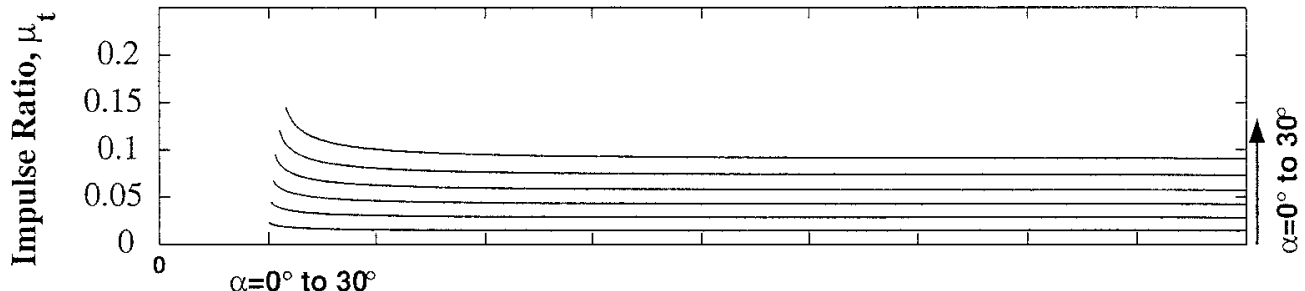

(b)

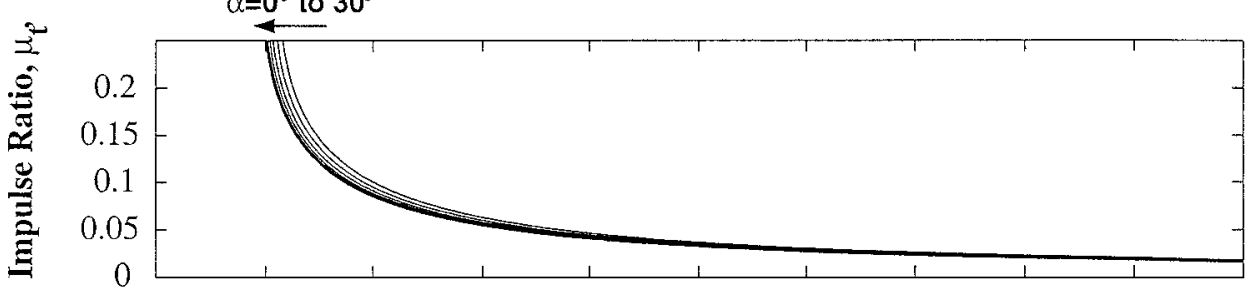

(c)



Figure 12. $e_{n}$ and $\mu$ for case No. 2.3 ( $\alpha=5^{\circ}$ increment). 
(a)

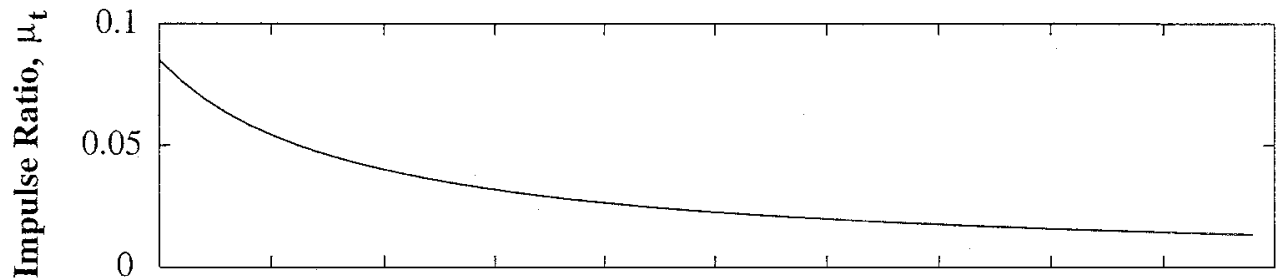

(b)

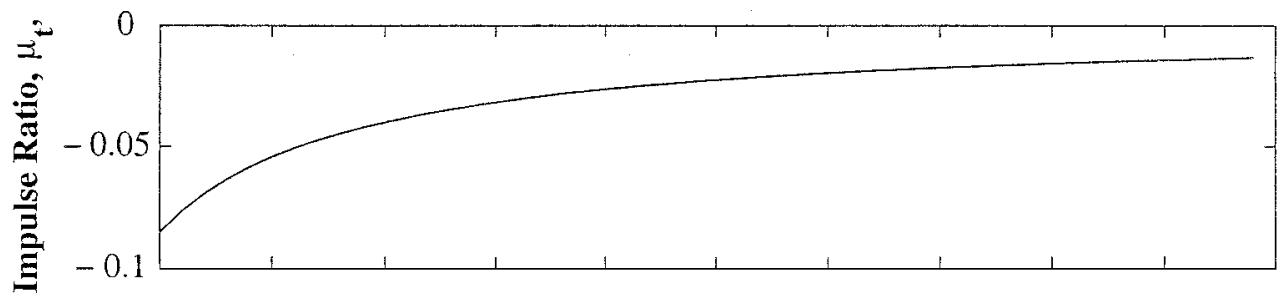

(c)

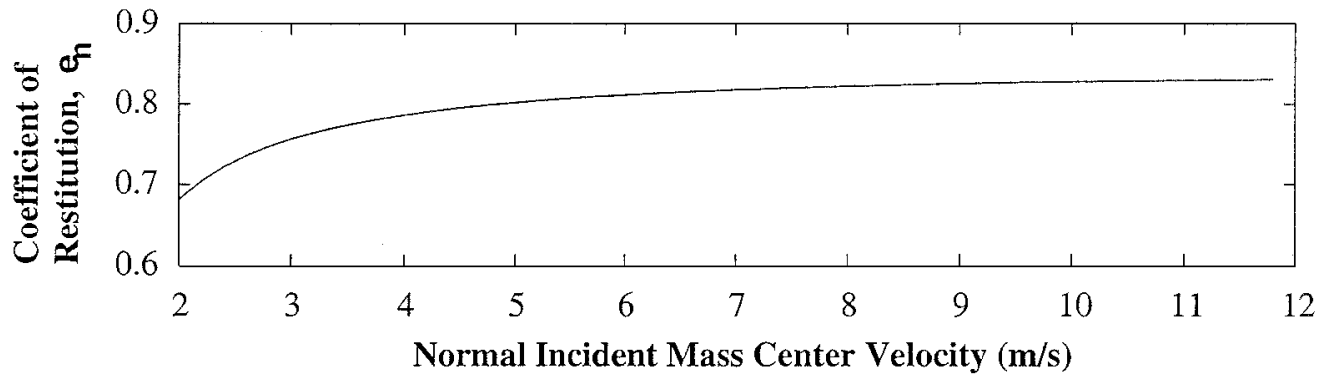

Figure 13. $e_{n}$ and $\mu$ for case No. 2.4 ( $f=0 \rightarrow 0.9$ increment).

(a)

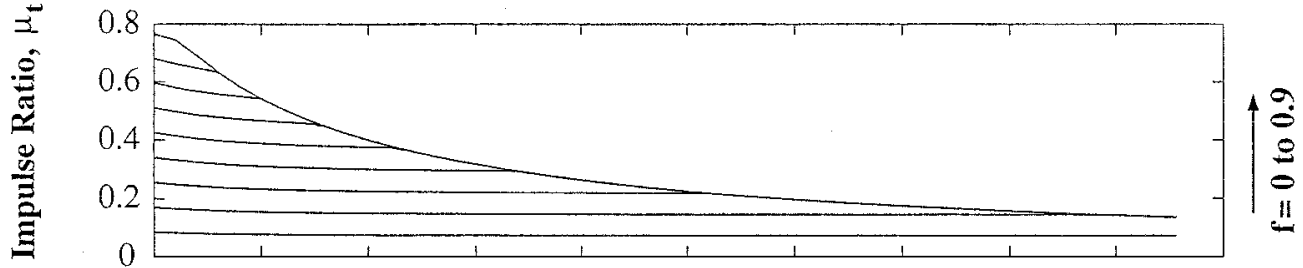

(b)



(c)

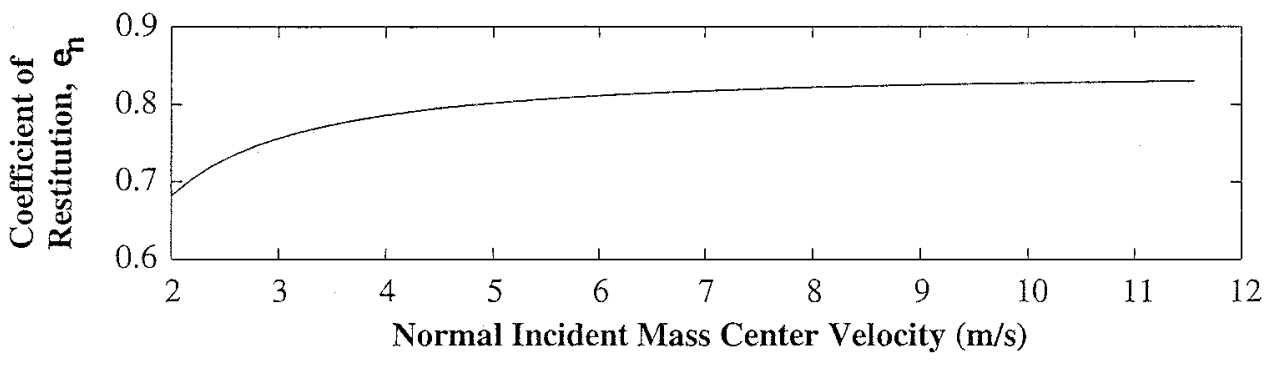

Figure 14. $e_{n}$ and $\mu$ for case No. 2.5 ( $f=0.1$ increment). 
is large. The effects on tangential impulse ratios in both $t$ and $t^{\prime}$ directions are significant and this is even more so for smaller normal impact velocities. For very low velocity and high spin impact or contact, the determination of friction coefficient is very important. On the other hand, in order to measure the friction coefficient accurately, the experiment should be conducted in the very low velocity and high rotational velocity range. The above-sensitivity analysis can be valuable in the design of the experiment of particle impact.

The results can be summarized as follows:

1. By changing the incident angle, $\alpha$, at low velocities, the impulse ratio, $\mu$, and the restitution coefficient, $e_{n}$, change significantly. The changes of the coefficient of restitution, $e_{n}$, at high velocities and energy loss, $K_{L}$ and $K_{t}$, are not as significant.

2. The effects of initial angular velocities are significant on all the calculations, including $\mu$ and energy losses. The effects are more significant for low initial velocities.

3. When the adhesion and damping forces increase, the particle is captured by the surface, followed by damped vibration with a decaying solution. On the contrary, rebound occurs for low adhesion and low damping cases.

\section{CONCLUSIONS}

This study clearly demonstrates that 3D simulations can provide important information for microsphere contact/impact. Through numerical simulation, the parameter sensitivity, properties of the forces, and factors that control the particle deposition and rebound are understood. The simulation shows that the rolling and translational energy transport plays an important role during attachment, including oblique impact. The results offer a better understanding of the friction and adhesion model. The tangential motion of a particle during impact is quite sensitive to the incident angles and the initial rotation.

The nonlinear analysis shows that any one of 3 types of motion can occur for a microsphere impact: rebound, capture with damped oscillation, and capture without oscillation. Which occurs is determined by the material parameters and the normal incident velocity. Two characteristic velocities, the capture velocity and the critical velocity, are defined in determining the conditions for capture and different types of motions after the capture, respectively. Capture occurs when the incident velocity of the microsphere is lower than the capture velocity such that the phase path of the microsphere falls into the sink on the phase portrait. The occurrence of oscillation after the capture is determined by the normal incident velocity and the critical velocity. If the normal incident velocity is smaller than the critical velocity, no oscillation occurs after the capture. The asymptotic frequency of the oscillation near the equilibrium position is determined by the material properties and the radius of the sphere. Because of the nonlinearity of the system, the asymptotic frequency is also dependent on the incident velocity if the damping coefficients, $C_{H}$ and $C_{A}$, have the form shown in Equations (15) and (14).

The simulation results also identify potential sources of errors in experiments and the best incident velocities for the measurement of different parameters. It is clear from the simulation results that experimental measurements of the rotational velocity of microspheres would greatly increase the understanding of low velocity oblique impacts. Analysis and experimental results show that some rolling models are not able to characterize rolling friction accurately. In order to simulate rolling resistance during established contact, the asymmetric distribution of the normal contact pressure over the contact area must be modeled properly. However, for impact problems, the rolling resistance can be neglected because the contact radius is very small and the duration of microsphere-substrate contact is very short.

\section{REFERENCES}

Ando, Y., and Ino, J. (1998). Friction and Pull-Off Forces on Submicron-Size Asperities, WEAR 216:115-122.

Ando, Y., Ishikawa, Y., and Kitahara, T. (1995). Friction Characteristics and Adhesion Force under Low Normal Load, Transactions of the ASME J. of Tribology 117:569-574.

Brach, R. M., and Dunn, P. F. (1995). Macrodynamics of Microparticles, Aerosol Sci. Technol. 23:51-71.

Brach, R. M., and Dunn, P. F. (1996). Simulation of Microparticle Impact with Adhesion, Proc. of Adhesion Soc., Symposium on Particle Adhesion, Myrtle Beach, S.C.

Brach, R. M., Dunn, P. F., and Cheng, W. (1999b). Rotational Dissipation During Microsphere Impact, Aerosol Sci. Technol. 30:1321-1329.

Brach, R. M., Dunn, P. F., and Li, X. (2000). Experiments and Engineering Models of Microparticle Impact and Deposition, J. Adhesion 74:227-282.

Burnham, N. A., Behrend, O. P., Oulevey, F., Gremaud, G., Gallo, P.-J., Gourdon, D., Dupas, E., Kulik, A. J., Pollock, H. M., and Briggs, G. A. D. (1997). How Does a Tip Tap?, Nanotechnol. 8:67-75.

Cheng, W., Brach, R. M., and Dunn, P. F. (2000). Numerical Studies on 3D Particle Impact With Surfaces, Hessert Center Technical Report, University of Notre Dame, Notre Dame, IN.

Cundall, P. A., and Hart, R. D. (1992). Numerical Modeling of Discontinua, Engineering Computations. 9:101-113.

Dahneke, B. (1975). Further Measurements of the Bouncing of Small Latex Spheres, J. Colloid Interface Sci. 51:58-65.

Derjaguin, B. V., Muller, V. N., and Toporov, Yu. P. (1975). Effect of Contact Deformations on the Adhesion of Particles, J. Colloid Interface Sci. 53:314325.

Dunn, P. F., Ibrahim, A., and Brach, R. M. (2001). Microparticle Resuspension Experiment, 20th Annual Meeting of the American Association for Aerosol Research, Portland, OR.

Gladwell, G. M. L. (1980). Contact Problems in The Classical Theory of Elasticity, Sijithoff \& Noordhoff, Alphen aan den Rijin.

Goldsmith, W. (1960). Impact-The Theory andPhysical Behaviourof Colliding Solids, Edward Arnold, London.

Hertz, H. (1881). Über die Beruhrung fester elastischer Korper, J. für die Reine und Angewandte Mathematik. 92:156-171.

Hertz, H. (1882). Über die Beruhrung fester elastischer Korper und Über die Harte, Verhandlungen des Vereins zur Beforderung des Gewerbfleifses, Berlin.

Israelachvili, J. N., and Tabor, D. (1972). The Measurement of van der Waals Dispersion Forces in the Range 1.5 to 130 nm, Proc. R. Soc. Lond. A 331:19 38.

Johnson, K. L. (1985). Contact Mechanics, Cambridge University Press, Cambridge. 
Johnson, K. L., Kendall, K., and Roberts, A. D. (1971). Surface Energy and the Contact of Elastic Solid, Proc. R. Soc. Lond. A 324:301-313.

Li, X., Dunn, P. F., and Brach, R. M. (1999). Experimental and Numerical Studies on the Normal Impact of Microspheres with Surfaces, J. Aerosol Sci. 30:439-449.

Ota, H., Oda, T., and Kobayashi, M. (1995). Development of Coil Winding Process for Radial Gap Type Electromagnetic Micro-Rotating Machine, Proc. IEEE MEMS 197-202.

Quesnel, D. J., Rimai, D. S., Gady, B., and DeMejo, L. P. (1998). Exploring the JKR Formalism with Finite Element Analysis, Proc. 21st Annual Meeting, Adhesion Society, Savannah, GA, pp. 290-292.

Rollot, Y., Regnier, S., and Guinot, J. (1999). Simulation of MicroManipulations: Adhesion Forces and Specific Dynamic Models, International J. Adhesion and Adhesives 19:35-48.

Savkoor, A. R., and Briggs, G. A. D. (1977). Effect of Tangential Force on the Contact of Elastic Solids in Adhesion, Proc. R. Soc. Lond. A 356:103-114.

Skinner, J., and Gane, N. (1972). Sliding Friction under Negative Load, J. Phys. D 5:2087-2094.
Thornton, C. (1991). Interparticle Sliding in the Presence of Adhesion, J. Phys. D 24:1942-1946.

Tsai, C. J., Pui, D. Y. H., and Liu, B. Y. H. (1991). Elastic Flattening and Particle Adhesion, Aerosol Sci. Technol. 15:239-255.

Wall, S., John, W., Wang, H. C., and Goren, S. L. (1990). Measurements of Kinetic Energy Loss for Particles Impacting Surfaces, Aerosol Sci. Technol. 12:926-946.

Wang, H. C. (1990). Effects of Inceptive Motion on Particle Detachment from Surfaces, Aerosol Sci. Technol. 13:386-393.

Xu, M. D., and Willeke, K. (1993). Right-Angle Impaction and Rebound of Particles, J. Aerosol Sci. 24:19-30.

Zhong, Z. (1993). Finite Element Procedures for Contact-Impact Problems, Oxford Science Publications, Oxford University Press Inc., New York.

Ziskind, G., Fichman, M., and Gutfinger, C. (1997). Adhesion Moment Model for Estimating Particle Detachment from a Surface, Aerosol Sci. Technol. 28:623-634.

Ziskind, G., Fichman, M., and Gutfinger, C. (2000). Particle Behavior on Surfaces Subjected to External Excitations, J. Aerosol Sci. 31:703-719. 